\title{
Selected wheat seed defense proteins exhibit competitive binding to model microbial lipid interfaces
}

Article

Accepted Version

Sanders, M. R., Clifton, L. A., Neylon, C., Frazier, R. A. and Green, R. J. (2013) Selected wheat seed defense proteins exhibit competitive binding to model microbial lipid interfaces. Journal of Agricultural and Food Chemistry, 61 (28). pp. 68906900. ISSN 1520-5118 doi: https://doi.org/10.1021/jf401336a Available at https://centaur.reading.ac.uk/33527/

It is advisable to refer to the publisher's version if you intend to cite from the work. See Guidance on citing.

To link to this article DOI: http://dx.doi.org/10.1021/jf401336a

Publisher: American Chemical Society

All outputs in CentAUR are protected by Intellectual Property Rights law, including copyright law. Copyright and IPR is retained by the creators or other copyright holders. Terms and conditions for use of this material are defined in the End User Agreement.

www.reading.ac.uk/centaur 
Central Archive at the University of Reading

Reading's research outputs online 
Selected Wheat Seed Defense Proteins Exhibit Competitive Binding to Model

Microbial Lipid Interfaces

Michael R. Sanders ${ }^{\dagger,}$, Luke A. Clifton ${ }^{\S}$, Cameron Neylon ${ }^{\S}$, Richard A. Frazier ${ }^{\ddagger}$ and Rebecca

J. Green*,†

${ }^{\dagger}$ Reading School of Pharmacy, University of Reading, PO Box 226, Whiteknights, Reading, RG6 6AP, UK

Department of Food and Nutritional Sciences, University of Reading, PO Box 226, Whiteknights, Reading, RG6 6AP, UK

${ }^{\S}$ ISIS Spallation Neutron Source, Science and Technology Facilities Council, Rutherford Appleton Laboratory, Harwell Science and Innovation Campus, Didcot, Oxfordshire, OX11 OQX, UK

*Corresponding Author (Tel: +44-118-3788446; Email: rebecca.green@reading.ac.uk) 


\section{ABSTRACT}

2 Puroindolines (Pins) and purothionins (Pths) are basic, amphiphilic, cysteine-rich wheat

3 proteins that play a role in plant defense against microbial pathogens. We have examined the

4 co-adsorption and sequential addition of Pins (Pin-a, Pin-b and a mutant form of Pin-b with

5 Trp-44 to Arg-44 substitution) and $\beta$-purothionin ( $\beta$-Pth) model anionic lipid layers, using a

6 combination of surface pressure measurements, external reflection FTIR spectroscopy and

7 neutron reflectometry. Results highlighted differences in the protein binding mechanisms,

8 and in the competitive binding and penetration of lipid layers between respective Pins and $\beta$ -

9 Pth. Pin-a formed a blanket-like layer of protein below the lipid surface that resulted in the

10 reduction or inhibition of $\beta$-Pth penetration of the lipid layer. Wild-type Pin-b participated in

11 co-operative binding with $\beta$-Pth, whereas the mutant Pin-b did not bind to the lipid layer in

12 the presence of $\beta$-Pth. The results provide further insight into the role of hydrophobic and

13 cationic amino acid residues in antimicrobial activity.

14

\section{KEYWORDS}

16 Antimicrobial peptide; puroindoline; purothionin; neutron reflectometry; FTIR spectroscopy;

17 surface pressure. 


\section{INTRODUCTION}

19 Plants produce proteins and peptides with antimicrobial and antifungal activities as a defense mechanism against pathogenic species, which exert their activity through interaction with the

21 cytoplasmic membrane of the target pathogen. ${ }^{1,2}$ In previous studies, we have characterized the lipid membrane interactions of puroindoline (Pin) and purothionin (Pth) proteins (both isolated from hexaploid wheat) using air/liquid monolayer membrane models. ${ }^{3-5}$ Pins are $\sim 13$ kDa proteins that occur as two wild-type isoforms, Pin-a and Pin-b, which both feature a Trprich domain that is thought to be the site of interaction with lipid membranes and has sequence similarity to indolicidin, a mammalian antimicrobial peptide. ${ }^{6}$ Pins are up-regulated during times of pathogenic attack and have been shown to act against known plant pathogens including fungal pathogens as well as Gram-positive and Gram-negative bacteria. ${ }^{7-9}$

The Trp-rich domain is not fully conserved between the wild-type isoforms; Pin-a contains five Trp residues (WRWWKWWK) and Pin-b has a truncated domain containing three Trp residues (WPTKWWK). ${ }^{10,11}$ Moreover, allelic variation in Pin-b gene expression within certain wheat varieties leads to a mutant form of Pin-b containing a single residue substitution of tryptophan to arginine (Trp-44 to Arg-44) within the Trp-rich domain. ${ }^{12}$ This Pin-b mutant domain has the sequence WPTKWRK and its presence in wheat is associated with the occurrence of hard-textured endosperm, which is a quality determinant for food use. ${ }^{13,14}$ Using a combination of surface-sensitive techniques, we have further demonstrated that this single residue substitution reduces depth of penetration into lipid membranes relative to the wild-type Pin-b, ${ }^{15,16}$ and we also determined a major effect of this point mutation on the synergistic interactions of Pin-a and Pin-b with respect to lipid membrane penetration. ${ }^{3}$

Pths are of lower molecular mass $(\sim 5 \mathrm{kDa})$ than the Pins and do not feature any Trp residues

41 within their primary structure. ${ }^{17}$ Here we focus on $\beta$-purothionin $(\beta$-Pth), which is believed to 
interact with lipids via a leucine-rich surface helix. ${ }^{18}$ The individual actions of Pths and Pins have been explored in vitro, where it has been established that they have contrasting mechanisms of action. ${ }^{19}$ They are co-localised in the wheat seed, which raises the possibility of synergistic or cooperative activity against pathogens. Here we examine interactions of Pin-

a, Pin-b (both wild-type and Trp-44 to Arg-44 mutant forms) and $\beta$-Pth as mixed and sequentially adsorbed systems with air/liquid lipid monolayer models so that we may test this hypothesis. Surface pressure measurements and external reflection-Fourier transform infrared (ER-FTIR) spectroscopy have been used to monitor the surface penetration and adsorption of mixed/sequential $\beta$-Pth/Pin systems to lipid monolayers. Although these techniques cannot differentiate between the different proteins within a system, the combined ability to probe the protein penetration and the lipid layer structure provided a useful insight into the mechanism of interaction of each protein with lipid membranes. In addition, neutron reflectometry (NR) has been employed to study the interfacial layer structure of selected systems.

\section{MATERIALS AND METHODS}

\section{Materials}

The anionic lipid, 1,2-dipalmitoyl-sn-glycero-3-phospho-(1'-rac-glycerol) (DPPG, synthetic, purity $>99 \%$ ), was purchased from Avanti Polar Lipids (Alabaster, AL, USA) and used without further purification. Stock solutions $(1 \mathrm{mg} / \mathrm{mL})$ of DPPG were prepared in HPLC grade chloroform (Sigma-Aldrich, Dorset, UK) and stored at room temperature. Wild-type Pin-a and Pin-b were extracted from flour milled from Claire winter wheat and purified using

63 Triton X-114 phase partitioning and chromatographic techniques as described previously. ${ }^{20}$

$64 \beta$-Pth was purified on a C18 reverse phase HPLC as described previously; ${ }^{21}$ the starting material used in this process was the Pth-rich fraction obtained as a by-product of the 
purification of Pin-b. The mutant Pin-bs was purified in the same manner but from flour milled from Soissons winter wheat (hence the designation as Pin-bs). Mixed protein solutions were prepared as 1:1 molar ratio to achieve the desired total protein concentration.

\section{Surface Pressure Measurements}

70

Surface pressure measurements were performed using a model 602m PTFE Langmuir trough (Nima Technology Ltd, Coventry, UK) equipped with barriers used for monolayer compression experiments. A paper Wilhelmy plate attached to a surface pressure sensor was used to monitor the surface pressure. Lipid monolayers were made at the air/liquid interface by a method described previously. ${ }^{22}$ Briefly, the trough was filled with $80 \mathrm{~mL}$ of $20 \mathrm{mM}$ sodium phosphate buffer (pH 7.0) and DPPG monomolecular layers were compressed and held in a condensed phase at $22 \mathrm{mN} / \mathrm{m}$. The stability of the lipid films was monitored through surface pressure vs. time measurements. When the lipid film had stabilized $1 \mathrm{~mL}$ of appropriate protein solution was added to the sub-phase so that the final concentration of the protein was $0.48 \mu \mathrm{M}$. Protein penetration into the lipid layer was then monitored as surface pressure vs. time measurements for approximately $120 \mathrm{~min}$ before addition of the second protein if studying sequential protein addition (total protein concentration in trough now at $0.96 \mu \mathrm{M})$. Protein binding was then monitored by surface pressure leading to a total protein adsorption time of $250 \mathrm{~min}$. Experiments were repeated three times to determine the mean change in surface pressure.

\section{External Reflection FTIR Spectroscopy}

ER-FTIR spectra were recorded using a ThermoNicolet Nexus instrument (Madison, WI, USA) fitted with a 19650 series monolayer/grazing angle accessory (Specac, Kent, UK). The instrument was also fitted with a mercury cadmium telluride detector and connected to an air dryer to purge the instrument of water and carbon dioxide. The accessory was also equipped 
with a small PTFE trough complete with a barrier used to control lipid compression; the grazing incident angle was aligned at $55^{\circ}$ to the surface of the trough. Access to the trough throughout the experiment was via a small sliding lid in order to maintain the dry air purge. Protein-lipid interactions were analyzed using external reflectance using a method described previously. ${ }^{22}$ All FTIR spectra were collected at a resolution $4 \mathrm{~cm}^{-1}$ where 256 interferograms were collected, co-added and ratioed against a background spectrum of $\mathrm{D}_{2} \mathrm{O}$ buffer solution.

In each experiment, $9.5 \mathrm{~mL}$ of $20 \mathrm{mM}$ sodium phosphate buffer prepared in $\mathrm{D}_{2} \mathrm{O}(\mathrm{pD} 7.0)$ was placed in the trough and a background single beam spectra was recorded allowing time for the sample chamber purge to remove $\mathrm{H}_{2} \mathrm{O}$ vapor and $\mathrm{CO}_{2}$ from the atmosphere. After recording a background spectrum, $5 \mu \mathrm{L}$ of $0.5 \mathrm{mg} / \mathrm{mL}$ DPPG was spread on to the surface of

100 the buffer and compressed to $22 \mathrm{mN} / \mathrm{m}$. Sample scans were taken after compression to ensure stability of the lipid film, which was monitored through the observation of the $\mathrm{CH}_{2}$ symmetric and asymmetric stretching frequencies in the phospholipid tails in the regions $2854-2850 \mathrm{~cm}^{-1}$ and $2924-2916 \mathrm{~cm}^{-1}$, respectively. Protein solution $(0.5 \mathrm{~mL})$ was injected into the sub phase in sequential experiments to make a final protein concentration of $0.48 \mu \mathrm{M}$ on addition of the first protein and a total subphase protein concentration of $0.96 \mu \mathrm{M}$ after

106 addition of both proteins. Spectra were continuously collected for the first 15 min after

107 protein injection, and one spectrum every $15 \mathrm{~min}$ for the rest of the collection time.

108 Sequential adsorption experiment timing was as described for surface pressure

109 measurements. The interaction of the protein with the lipid monolayer was observed by

110 monitoring the amide I region, $1700-1600 \mathrm{~cm}^{-1}$ and the aforementioned $\mathrm{CH}_{2}$ asymmetric and 111 symmetric stretching frequencies.

112 To correct for any water vapor present $\mathrm{H}_{2} \mathrm{O}$ and HOD spectra were scaled and subtracted 113 against protein adsorbed spectra, the degree of subtraction was dependent on the adsorption 
114 time as well as the amount of H/D exchange. The HOD spectra used for scaling and

115 subtraction purposes were collected during the purge of the sample area prior to the addition

116 of the lipid film. No further processing was performed to the data. Experiments were

117 performed in triplicate unless stated otherwise

\section{Neutron reflectivity of Pin-a and B-Pth synergistic systems}

119 Neutron reflectivity (NR) datasets were collected and reduced at SURF and CRISP neutron

120 reflectometers at ISIS (Rutherford Appleton Laboratory, Didcot, UK) using respective $Q$

121 ranges of $0.01-0.35$, which translates to neutron wavelengths of 0.55-6.8 $\AA$ and 0.5-6.5 $\AA$

122 respectively. Neutron scattering is a nuclear effect such that for hydrogen and deuterium the

123 scattering length is significantly different (Table 1), which allows the use of isotopic

124 substitution to produce a number of reflectivity profiles corresponding to a single interfacial

125 structure. ${ }^{23}$ In conjunction with NR, this provides a way of identifying the interfacial

126 structure of a multicomponent system. Details of the procedure to obtain and fit protein-lipid

127 profiles have been described previously. ${ }^{5}$

128 Protein adsorption to DPPG monolayers was measured on a PTFE Langmuir trough as

129 described above for surface pressure measurements. NR profiles were recorded before and

130 after addition of protein, allowing time for equilibrium of the lipid/protein systems.

131 Experiments were carried out on an aqueous subphase composed of air contrast matched

132 water (non-reflective water (NRW): $8 \% \mathrm{D}_{2} \mathrm{O}, 92 \% \mathrm{H}_{2} \mathrm{O}$ ); this was to make the reflectivity

133 profile sensitive only to material at the air/liquid interface. Data was collected at two angles

134 for experiments on NRW $0.7^{\circ}$ and $1.5^{\circ}$; the beam intensity was calibrated with respect to a

135 clean $\mathrm{D}_{2} \mathrm{O}$ surface. Data was obtained using phospholipids with hydrogenated and deuterated

136 tail regions to provide isotopic contrast between the protein and the phospholipid at the

137 interface. 
138 The raw data from NR experiments was reduced and data from multiple angles was stitched

139 together at the respective beamline. The reflectivity profiles were then analyzed using optical

140 matrix formalism, ${ }^{24}$ to fit Abeles layer models to an interfacial structure using the data-fitting

141 program RasCAL developed at ISIS by A. Hughes. A typical modeling procedure calculates

142 the reflectivity based on fitting structural parameters; number of layers at the interface,

143 thickness $(\tau)$ and scattering length density $(\rho)$ of each layer and layer roughness. A set of

144 reflectivity profiles measured under different isotopic conditions are fitted together to the

145 same parameters except for differences in scattering length density; this allows different

146 components within the system to be highlighted, and the volume fraction $(\Phi)$ of each

147 component to be determined. ${ }^{25}$

148 For each layer within the fit, the scattering length densities of the individual components

149 (Table 1) can be multiplied by their respective volume fractions to give the measured

150 scattering length density for each isotopic contrast reflectivity profile. Thus, the volume

151 fraction of each component within each interfacial layer can be determined. For mixed

152 protein systems, the scattering length density was calculated as the average of Pin-a and $\beta$ -

153 Pth. ${ }^{26,27}$ The surface area and the surface excess are calculated directly from the calculated

154 volume fractions. With knowledge of the volume fraction of each component at the interface,

155 the area per molecule and surface excess can be calculated assuming that the surface is made

156 of uniform layers. ${ }^{28}$

157 For the Pin-a/ $\beta$-Pth systems a three-layer model was needed to provide a suitable fit of the

158 data; this model comprised of two layers to describe the tail and head regions of the lipid

159 layer, and a third layer showing presence of protein below the lipid layer. Fitting was

160 constrained to the assumption that the lipid molecules are arranged such that the first lipid

161 layer contains the lipid tails while the second layer contains lipid head groups. Experimental 
data fitting errors were carried out as described previously using RasCAL's "bootstrap" error analysis function. ${ }^{5}$

\section{RESULTS AND DISCUSSION}

\section{Co-adsorption of $\beta$-Pth and Pin proteins at an anionic lipid surface}

166 Surface pressure measurements and ER-FTIR spectroscopy were used to probe lipid

167 penetration and the relative mass of protein adsorbed at the lipid interface both in and below

168 the lipid layer. ${ }^{24}$ Figure 1 shows surface pressure versus time and amide I peak area versus

169 time for the binding of mixed $\beta$-Pth/Pin protein systems to a DPPG condensed monolayer

170 from total protein solution concentrations of $0.48 \mu \mathrm{M}$ and $0.96 \mu \mathrm{M}$. Values for surface

171 pressure change and amide I peak area are given in Table 2, where the mixed protein systems

172 are compared with values for lipid binding of the individual proteins.

173 Figure 1A reveals significant differences for each of the $0.48 \mu \mathrm{M}$ mixed protein systems with

174 respect to the surface pressure increase upon binding to the DPPG layer. For the $\beta$-Pth/Pin-a

175 mixture, the surface pressure increased over the first $50 \mathrm{~min}$ before equilibrating at approximately $29 \mathrm{mN} / \mathrm{m}$, which represented an increase of $7.3 \pm 0.8 \mathrm{mN} / \mathrm{m}$. For $\beta$-Pth/Pin-bs

177 the increase in surface pressure was equivalent to that for the $\beta$-Pth/Pin-a system; however, the rate of increase was slower. The $\beta$-Pth/Pin-b mixed system resulted in a significantly lower increase in surface pressure of only $3.2 \pm 0.3 \mathrm{mN} / \mathrm{m}$. These results for the mixed

180 protein systems revealed differences in the level of penetration of protein into the lipid layer that could not be directly related to the surface pressure values recorded for Pin proteins binding as single proteins. This particularly relates to the $\beta$-Pth/Pin-b mixed system. The

183 surface pressure change for Pin-b penetration was the highest of the three Pin proteins and binding of $\beta$-Pth as a single protein also resulted in a similar high level of lipid penetration 
185 (Table 2). However, for the mixed system there was observed a significant reduction in 186 penetration to approximately one-third the level for either of the individual proteins. This 187 could not be ascribed to a concentration effect, since $0.24 \mu \mathrm{M}$ Pin-b, which equates to the 188 concentration of Pin-b present in the $0.48 \mu \mathrm{M}$ mixed protein system, results in a surface 189 pressure shift of $9.0 \mathrm{mN} / \mathrm{m}$ (data not shown).

190 The FTIR spectra provided further information about the protein-lipid interactions through 191 changes in the carbonyl and amide I region (1800 and $\left.1550 \mathrm{~cm}^{-1}\right)$ and the hydrocarbon 192 region, particularly the $\mathrm{C}-\mathrm{H}$ stretch region between 3050 and $2750 \mathrm{~cm}^{-1}$. In the $\mathrm{C}-\mathrm{H}$ stretch 193 region, the $\mathrm{CH}_{2}$ asymmetric stretch at approximately $2920 \mathrm{~cm}^{-1}$ was monitored to investigate 194 formation of the compressed lipid monolayer and the effect of protein addition on the lipid layer structure. Within the carbonyl region, a peak at $1735 \mathrm{~cm}^{-1}$ was observed corresponding to the C-O stretch vibration within the lipid head group, and a peak at approximately 1650

$197 \mathrm{~cm}^{-1}$ was observed corresponding to the protein amide I peak.

198 For the $\beta$-Pth/Pin co-adsorption experiments, no change in the lipid hydrocarbon peaks was observed during protein binding to the lipid surface; however, on addition of protein, the amide I peak was present and its peak area monitored as a function of time. Figure 1B shows

201 the change in the amide I peak during the adsorption of the $0.48 \mu \mathrm{M}$ mixed protein systems to the condensed phase DPPG at the air/liquid interface. As observed for the surface pressure data, the FTIR data for the mixed systems does not quantitatively match or fit to a pattern that might be suggested by the behavior of the individual Pin proteins. For example, the amide I

205 peak area change for $0.48 \mu \mathrm{M} \beta$-Pth/Pin-bs suggested a similar level of binding to that of $0.48 \mu \mathrm{M} \beta$-Pth, but not to $0.48 \mu \mathrm{M}$ Pin-bs (Table 2). This observation, together with the surface pressure data may suggest some level of competitive adsorption from these mixed system solutions. 
209 At the higher protein concentration of $0.96 \mu \mathrm{M}$, differences in adsorption behavior between

210 the $\beta$-Pth/Pin systems were less obvious (Table 2). With regard to surface pressure

211 measurements, all protein systems resulted in a rapid increase in surface pressure that reached

212 equilibrium within 10 min leading to a surface pressure change of $9.9,11.3$ and $13.7 \mathrm{mN} / \mathrm{m}$

213 for $\beta$-Pth/Pin-a, $\beta$-Pth/Pin-b and $\beta$-Pth/Pin-bs systems, respectively. The rate of increase in

214 surface pressure was similar to that measured for $\beta$-Pth binding alone. ${ }^{5}$ FTIR data showed a

215 rapid appearance and then increase in the amide I peak area for adsorption of $\beta$-Pth/Pin-a and

$216 \beta$-Pth/Pin-b to the lipid surface. As shown in Table 2, the peak area increased to values

217 similar to those observed for the single protein Pin systems at concentration of $0.48 \mu \mathrm{M}$. For

$218 \beta$-Pth/Pin-bs, the FTIR peak area isotherm is different, showing two rates of adsorption; an

219 initial rapid increase (to a peak area of approximately 0.05 ) that begins to plateau before a

220 second increase in peak area at approximately $50 \mathrm{~min}$ to reach equilibrium. The final peak

221 area was similar to that of the Pin-bs only system. This appears to suggest initial adsorption

222 or penetration of the smaller $\beta$-Pth before blanket like adsorption of Pin-bs. Thus, at the

223 higher protein concentration $(0.96 \mu \mathrm{M})$ of the mixed system, Pin-bs was more competitive

224 compared to binding at lower concentrations $(0.48 \mu \mathrm{M})$ where $\beta$-Pth dominated.

225 These results show protein concentration dependence of the competitive binding behavior to the lipid surface particularly for systems involving Pin-b and Pin-bs. For Pin-a, adsorption

227 reaches values similar to $0.48 \mu \mathrm{M}$ Pin-a only for the mixed $\beta$-Pth/Pin-a $(0.48 / 0.48 \mu \mathrm{M})$

228 sample, however penetration, as seem by surface pressure measurements, is greater and more

229 like that seen for $0.48 \mu \mathrm{M} \beta$-Pth. $\beta$-Pth/Pin-b shows depressed levels of penetration and

230 binding at the lower concentration. However, when the concentration is increased, both the

231 levels of lipid penetration and adsorption of protein below the film are enhanced relative to 

the individual proteins. Similarly, Pin-bs was shown to compete with $\beta$-Pth rather poorly at

233 lower concentration compared to when the total protein concentration is increased.

234 For the FTIR adsorption experiments, differences in the shape of the amide I peaks provided 235 information on the dominant secondary structure of the adsorbed protein and the lipid 236 surface. Figure 2 shows the carbonyl region of the spectra for the co-adsorption of each 237 mixed protein system at $0.96 \mu \mathrm{M}$, and also shows deconvolution of the amide I peak. The 238 deconvoluted amide I peaks of the three Pin proteins have been reported previously, ${ }^{3,16}$ while 239 others have reported that $\beta$-Pth has a high helical content in contact with lipid. ${ }^{29}$ For each of 240 the protein systems, $\beta$-Pth/Pin-a, $\beta$-Pth/Pin-b and $\beta$-Pth/Pin-bs, the amide I peak shape after

24115 min adsorption was similar showing a symmetrical peak centered at approximately 1644

$242 \mathrm{~cm}^{-1}$. Deconvolution of these peaks enables contributions of different secondary structure environments to be compared between the spectra, and shows a split in the amide I peak that suggests some $\beta$-sheet content (at approximately $1680 \mathrm{~cm}^{-1}$ and $1620 \mathrm{~cm}^{-1}$ ), high helix content $\left(1655 \mathrm{~cm}^{-1}\right)$ and random coil $\left(1640 \mathrm{~cm}^{-1}\right)$. The deconvoluted spectra show that upon adsorption reaching equilibrium, after $60 \mathrm{~min}$, the random coil content of the adsorbed protein layer dominates for the $\beta$-Pth/Pin-b and $\beta$-Pth/Pin-bs systems but not for $\beta$-Pth/Pin-a. This can also be observed in the raw spectra, where the peak maximum shifts towards 1640 $\mathrm{cm}^{-1}$ during lipid binding. From our knowledge of the secondary structure of these proteins, 250 this shift towards higher random coil structure would be consistent with an increase in the amount of Pin-b or Pin-bs at the interface. Indeed, the deconvoluted spectra of $\beta-\mathrm{Pth} / \mathrm{Pin}-\mathrm{b}$ and $\beta$-Pth/Pin-bs after 60 min adsorption are remarkably similar to those observed for Pin-b and Pin-bs alone. ${ }^{16}$ According to our previous studies, Pin-a appears to have a higher helix content compared to Pin-b in the presence of lipid and, therefore, less change would be expected for competitive adsorption between Pin-a and $\beta-P$ th. ${ }^{3}$ 


\section{Sequential protein adsorption to an anionic lipid surface}

257 Co-adsorption experiments provided evidence of a competitive nature to protein binding to

258 the lipid surfaces. However, if one protein was associated with the lipid first, would this

259 impact on the lipid binding behavior of subsequent adsorption of a second protein? To answer

260 this, experiments have been carried out on sequential protein adsorption experiments of $\beta$-Pth

261 and Pins to a condensed DPPG monolayer at the air-liquid interface. The surface pressure

262 profiles and amide I peak areas are shown in Figure 3; values for surface pressure change

263 upon protein addition to the condensed lipid layer are given in Table 3, and for amide I peak

264 areas in Table 4.

265 In Figure 3A, $0.48 \mu \mathrm{M} \beta$-Pth was added to the buffer subphase and the surface pressure monitored for approximately 120 min before addition of $0.48 \mu \mathrm{M}$ of either Pin-a or Pin-b.

267 Figure $3 \mathrm{C}$ shows the surface pressure profiles for sequential adsorption where the Pin protein is added first. From Figure 3A it can be observed that upon the addition of $\beta$-Pth to the subphase there was a rapid increase in the surface pressure within the subsequent ten minutes.

270 The system had fully equilibrated to give an increase of $9.5 \pm 0.6 \mathrm{mN} / \mathrm{m}$ before the addition of the second protein after 120 min (Pin-a or Pin-b). Upon addition of Pin-a to a preadsorbed $\beta$-Pth system, the surface pressure quickly increased by $1.6 \pm 0.3 \mathrm{mN} / \mathrm{m}$ within 30 minutes and then equilibrated; the total surface pressure change of the complete system was $11.1 \pm$ $0.4 \mathrm{mN} / \mathrm{m}$. When Pin-b was added to a preadsorbed $\beta$-Pth system, there was a negligible increase in surface pressure, with a total surface pressure change for the complete $\beta-\mathrm{Pth} / \mathrm{Pin}-\mathrm{b}$ system of $9.4 \pm 0.5 \mathrm{mN} / \mathrm{m}$ as compared to $9.0 \pm 0.8 \mathrm{mN} / \mathrm{m}$ for $\beta$-Pth alone.

277 When the order of the protein addition is reversed, Pin-a and Pin-b show slower kinetics towards equilibrium binding than those for $\beta$-Pth, giving a surface pressure increase of $7.9 \pm$ 
work. ${ }^{5,16}$ On the addition of $\beta$-Pth to a preadsorbed Pin-a system there was a rapid increase in surface pressure, equilibrating at a total surface pressure change for adsorption of both proteins (total protein concentration of $0.96 \mu \mathrm{M}$ ) at $9.3 \pm 0.3 \mathrm{mN} / \mathrm{m}$. This total surface pressure change is similar to the surface pressure change for the $0.48 \mu \mathrm{M} \beta$-Pth single protein system on this trough (Figure 3A). When $\beta$-Pth was added to a preadsorbed Pin-b/DPPG layer, a small increase was observed giving a total pressure change for both proteins of $10.2 \pm$ $0.6 \mathrm{mN} / \mathrm{m}$.

287 Comparison of the surface pressure changes for these sequential adsorption systems shows similar total surface pressure changes after adsorption of the two proteins between all systems, ranging from 9.3 to $11.1 \mathrm{mN} / \mathrm{m}$, and using the Bonferroni multiple comparison $(\mathrm{P}<0.05)$ statistical test there are no significant differences between the different systems where the Pins were added first; however, the changes are significant when the $\beta$-Pth is added to the subphase first and followed by Pin-a. Furthermore, there are differences in the step changes on addition of the second protein highlighting differences in the ability of the individual proteins to penetrate into the lipid layer. Since surface pressure changes are sensitive to penetration of protein into the lipid layer, a limit in the maximum increase in surface tension at high protein concentration might be expected upon full compression of the 297 lipid layer.

The amide I peak areas from the ER-FTIR experiments for these sequential adsorption systems are shown in Figure 3B and D. The associated spectra showing the carbonyl region both prior to addition of protein and after adsorption equilibrium of each sequentially

301 adsorbed protein are given in Figure 4. Figure 3B compares the two sequential systems where $302 \beta$-Pth was added to the lipid subphase first and Pin-a or Pin-b was added second. Upon $\beta$-Pth addition, adsorption of protein was observed by the rapid appearance of a peak in the amide I 
region to produce a peak maximum at $1644 \mathrm{~cm}^{-1}$; the system was fully equilibrated within 10 minutes after protein addition. Upon the addition of Pin-a to the $\beta$-Pth/lipid system the size of

306 the amide I peak increased four-fold within $10 \mathrm{~min}$ and the system fully equilibrated within

307 an hour with a peak maximum at $1643 \mathrm{~cm}^{-1}$. Addition of Pin-b to the $\beta$-Pth adsorbed lipid

308 surface resulted in a two-fold increase in the amide I peak area and a shift in the peak

309 maximum to $1640 \mathrm{~cm}^{-1}$. The corresponding final peak area values are given in Table 4 .

310 When the order of protein addition was reversed, the addition of Pin-a to the DPPG layer was

311 accompanied by the appearance of a strong peak in the amide I region with a peak maximum

312 at $1644 \mathrm{~cm}^{-1}$ (Figure 3B and 4). According to the differences observed in the amide I peak

313 areas, the amount of Pin-a adsorbed at $0.48 \mu \mathrm{M}$ was approximately four-times that of $\beta$-Pth to

314 DPPG and equivalent to the total protein adsorption (at $0.96 \mu \mathrm{M}$ ) for $\beta$-Pth/Pin-a sequential

315 adsorption. This can be seen from comparison of peak area data in Tables 2 and 4. Addition

316 of $\beta$-Pth to the Pin-a/DPPG surface resulted in no further increase in adsorbed amount

317 according to the amide I peak area.

318 When Pin-b is added to the sub phase first (Figure 3D), the amide I peak area reaches a value

319 of approximately 0.075 at equilibrium; this value is approximately half that observed for

320 adsorption of Pin-a and equivalent to the value seen for the total adsorption of the $\beta-P$ th/Pin-b

321 sequential system. Addition of $\beta$-Pth to the Pin-b/lipid surface led to an increase in the amide

322 I peak area from 0.075 to 0.93 , resulting in a final amide I peak area that was $30 \%$ greater

323 than the total amide I peak area observed when $\beta$-Pth is adsorbed to the lipid layer first.

324 Regarding the impact that the protein binding has on lipid structure, our data (not shown)

325 supports previous reports, ${ }^{5}$ with a $\sim 8 \%$ reduction in $\mathrm{CH}_{2}$ asymmetric peak area upon $\beta$-Pth

326 addition. However, this only occurs in cases where $\beta$-Pth is adsorbed first. If added to a pre-

327 adsorbed Pin/lipid surface the purothionin is not able to disrupt the lipid surface. Thus the 
mechanism of lipid removal as suggested in the literature is prevented or reduced in the presence of puroindolines. ${ }^{19,30}$

330 For the $0.96 \mu \mathrm{M}$ Pin-b/ $\beta$-Pth system, the amide I peak maximum shifted towards $1640 \mathrm{~cm}^{-1}$

331 during adsorption, suggesting a change in secondary structure of the adsorbed protein

332 towards an increase in random coil, seen from deconvolution of the amide I peak. The 333 observed shift in the amide I peak appears to link with an increase in the amount of random

334 coil correlating with an increase in the amount of Pin-b at the interface. ${ }^{3,16}$ Clearly, the adsorption here is competitive, with Pin-b appearing to dominate at equilibrium. This finding is reflected when the proteins are added sequentially to the lipid, where we see evidence of

337 greater adsorption (amide I peak area) and penetration (surface pressure change) of protein into lipid when Pin-b is added first. If $\beta$-Pth is first these values are reduced compared to when the proteins are co-adsorbed.

\section{NR analysis of the protein-lipid layer structure for the co-adsorbed protein systems}

341 To determine the protein-lipid layer structure for protein binding to the lipid monolayer, neutron reflectivity studies have been carried out to compare the lipid binding behavior of the $\beta-$ Pth/Pin-a co-adsorbed and sequential binding systems. This enabled us to confirm levels of penetration compared to binding and adsorption below the lipid layer, and to compare with the pure protein adsorption studies reported previously. ${ }^{5}$

346 Figure 5A shows the NR profile and the best NR model to data fit obtained from a monolayer

347 of tail deuterated DPPG at the air/liquid interface compressed to $22 \mathrm{mN} / \mathrm{m}$ on a NRW

348 subphase. The scattering length density profile across the interface that is described by the fit

349 is shown in Figure 5B, and the structural parameters obtained from these fits are given in

350 Table 5. The phospholipid layer was fitted to a two-layer model, where thicknesses of the lipid acyl region and lipid head group were $16.4 \AA$ and $6.3 \AA$ respectively. A volume fraction 
$352\left(\Phi_{\text {lipid acyl }}\right)$ of 0.91 was calculated for the DPPG acyl chain in the condensed phase with an area 353 per molecule of $54.1 \AA^{2}$.

354 Figure 6A shows the NR profiles and the best three layer fit obtained for Pin-a/ $\beta$-Pth 355 coadsorbed $(0.96 \mu \mathrm{M})$ to a condensed phase DPPG monolayer; the scattering length density 356 profile of the fit is shown in Figure 6B, and the resulting structural parameters obtained are 357 given in Table 5. The best model-to-data fit used a three layer interfacial structure, where 358 layer 1 and 2 represented the lipid acyl chain and the head group regions of the phospholipid 359 respectively, and layer 3 represented the protein in the subphase below the lipid layer. The 360 layers were found to be $17.3,8$ and $37 \AA$ A respectively. Protein was found to have penetrated 361 the lipid layer and uniformly distributed within the acyl and lipid head group regions $\left(\Phi_{\text {protein }}\right.$ $362=0.16$ and 0.13 , respectively). The protein volume fraction below the lipid layer was found to 363 be 0.36 . Calculation of the protein surface excess showed a total amount of protein of 2.18 $364 \mathrm{mg} / \mathrm{m}^{2}$ where $78 \%\left(1.72 \mathrm{mg} / \mathrm{m}^{2}\right)$ was found underneath the lipid layer and $14 \%\left(0.32 \mathrm{mg} / \mathrm{m}^{2}\right)$ 365 was found in the acyl region. The protein surface excess and the thickness of the protein layer 366 showed similarities with the values previously observed when Pin-a at $0.48 \mu \mathrm{M}$ was adsorbed

367 to DPPG alone, ${ }^{5}$ both showing a protein layer thickness of approximately $34 \AA$ and similar 368 amounts of total protein surface excess (Table 5). The main differences observed were greater 369 penetration of protein into the acyl region of the lipid and a reduced amount of protein within 370 the head group region for the $\beta$-Pth/Pin-a system, signifying a difference in the depth of 371 penetration of protein into the lipid as a result of the presence of $\beta$-Pth. Previous studies 372 showing the binding of $\beta$-Pth to DPPG at $0.48 \mu \mathrm{M}$ showed penetration into the acyl lipid 373 region to be $0.6 \mathrm{mg} / \mathrm{m}^{2}$, with $0.31 \mathrm{mg} / \mathrm{m}^{2}$ within the head group and only $0.36 \mathrm{mg} / \mathrm{m}^{2}$ within a $3749 \AA$ A region below the lipid layer. ${ }^{5}$ 
375 Figures $6 \mathrm{C}$ and $6 \mathrm{D}$ show the NR profile, the best three layer fit and the resulting scattering

376 length density profile for the sequential protein adsorption experiments where $0.48 \mu \mathrm{M} \beta$-Pth

377 is adsorbed to a DPPG surface with pre-adsorbed Pin a $(0.48 \mu \mathrm{M})$. The structural parameters

378 for the three-layer fit are shown in Table 5. As with the coadsorbed film, the best model-to-

379 data fit obtained for the sequential addition of $\beta$-Pth adsorbed to a Pin-a/DPPG surface was a

380 three layer interfacial structure with layer thicknesses of $20 \AA, 10 \AA$ and $34 \AA$ for the lipid

381 acyl chain, lipid head group and protein below the film respectively. The volume fraction of

382 lipid was shown to decrease on addition of protein to the lipid surface due to an increase in

383 lipid layer thickness from 22.7 to $30 \AA$. The layer before adding $\beta$-Pth was a Pin-a/DPPG

384 layer that has been described previously as having a lipid layer thickness of $26 \AA$ and a

385 protein layer below the lipid of $33.5 \AA$; the distribution of protein between these layers was

$3860.2,0.51$ and $1.55 \mathrm{mg} / \mathrm{m}^{2}$, respectively. ${ }^{5}$ Table 5 shows that on addition of $0.48 \mu \mathrm{M} \beta$-Pth to

387 this system, the lipid layer became thicker and the amount of protein within the acyl lipid

388 region and below the lipid layer increased by $0.25 \mathrm{mg} / \mathrm{m}^{2}$ and $0.26 \mathrm{mg} / \mathrm{m}^{2}$, respectively.

389 The NR data supports the findings from FTIR experiments that showed Pin-a as the dominant

390 protein adsorbed from mixed $\beta$-Pth/Pin-a systems. However, the presence of a pre-adsorbed

391 Pin-a layer does not prevent a small increase in surface pressure on addition of $\beta$-Pth $(1.6 \pm 1$

$392 \mathrm{mN} / \mathrm{m}$ ), which was indicative of additional penetration of protein into the lipid. This was

393 confirmed by NR where sequential addition of $\beta$-Pth to a Pin-a/lipid surface resulted in

394 increased protein within the lipid head and tail regions and an increased thickness of the lipid

395 layer from $23 \AA$ for the pure lipid layer to $26-27 \AA$ after addition of Pin-a or a mixed Pin-a/ $/ \beta$ -

396 Pth solution to $30 \AA$ after sequential adsorption of the two proteins. Although lipid penetration

397 was enhanced compared to Pin-a only, NR data of the mixed and sequential adsorbed Pin-

$398 \mathrm{a} / \beta$-Pth systems showed less penetration into the lipid tail region to that seen for lipid binding 
399 of $\beta$-Pth only, ${ }^{5}$ showing that Pin-a has apparently hindered the lipid penetrative behavior of

$400 \quad \beta$-Pth.

\section{Competitive binding between $\beta$-Pth and Pins}

402 We have examined the possibility of a synergistic mechanism of interaction of the proteins $\beta$ -

403 Pth and Pins with respect to their lipid binding properties. However, data have not shown

404 evidence of strong synergy in binding behavior where the presence of the two proteins might

405 lead to enhanced lipid binding. Indeed, competitive binding behavior and differences in the

406 mode of lipid binding of the two types of proteins have been observed.

407 FTIR and NR measurements from this study and previous studies have shown that the Pins

408 form a thick protein layer below the lipid surface of approximately $35 \AA .^{5,15,16}$ In contrast the

409 total adsorbed amount for $\beta$-Pth is much less as shown by the peak area of the amide I peak

410 by FTIR and in previous studies by NR measurements. ${ }^{5}$ However, the relatively small size (5

$411 \mathrm{kDa}$ ) and helical amphipathic structure of $\beta$-Pth enables it to more rapidly penetrate into the

412 lipid layer. It is less hydrophobic than any of the Pins, but highly cationic with a charge of +8

413 at $\mathrm{pH} 7$.

414 The lipid binding Trp-rich loop of the different Pin proteins differs by the number of Trp

415 residues, but the Pins have similarities in MW, hydrophobicity and isoelectric points. Pin-a

416 has a pI of 10 and Pin-b has a pI of 11 according to $2 \mathrm{D}$ electrophoresis studies. ${ }^{31}$ However,

417 Pin-b is recognised to be more water-soluble than Pin-a and less inclined to self-associate in

418 aqueous solution; ${ }^{32}$ at $\mathrm{pH} 7$ its net charge is +9 compared to +6 for Pin-a. The difference in

419 behavior of the Pin proteins appears to be associated with the Trp-rich loop, rather than total

420 charge or hydrophobicity of the proteins; however, the behaviour is not simply linked to

421 number of Trp or cationic residues in this loop. Pin-b is the more penetrative in terms of lipid

422 binding of the Pins with three Trp residues within the loop, compared to five for Pin-a and 
two for Pin-bs. However, it does have two proline residues within the loop and fewer charged residues within that region, which may promote deeper penetration into the hydrophobic region of the lipid layer, thus behaving most like $\beta$-Pth in terms of lipid-penetration. Both Pin-a and Pin-bs adsorb strongly to the lipid head group region and penetrate less into the lipid tail region of the lipid layer. Pin-a however, competes very well with $\beta$-Pth and appears to dominate at the lipid surface, whereas Pin-bs competes very poorly and is prevented from binding strongly to the lipid in the presence of $\beta$-Pth.

Substituting Pin-b for Pin-bs results in significant differences in the lipid binding behavior of the mixed protein systems studied here, and highlights the impact that the amino acid sequence within the Trp-rich loop. The difference between the proteins is a point mutation substitution of Trp to Arg that alters the Trp-rich domain sequence from WPTKWWK for Pin-b to WPTKWRK for Pin-bs. This Trp to Arg substitution has been shown previously to reduce the lipid penetrative ability of the protein whilst enhancing association below the lipid

436 film, through interaction with the head group of the lipid. ${ }^{15}$ Upon co-adsorption of Pin-bs with $\beta$-Pth, $\beta$-Pth dominated at low protein concentrations and prevented binding of Pin-bs. $\beta$-Pth also dominated initially over Pin-bs at the higher concentration studied ( $0.96 \mu \mathrm{M})$, as evidenced by a two-step adsorption profile (Figure 1D). However, Pin-b and Pin-bs were shown to dominate lipid binding at equilibrium at high concentration $(0.96 \mu \mathrm{M})$ as observed from the changes to the FTIR amide I peak during adsorption (Figure 2).

The poor ability of Pin-bs to compete with $\beta$-Pth especially at low concentrations is particularly interesting and, when compared to Pin-b, highlights the importance of the

444 hydrophobicity of the lipid-binding region of the protein. The findings also link to our 445 previous studies where the co-binding of Pin-a and Pin-b to lipids was investigated and 446 revealed reductions in lipid penetration and binding when Pin-bs was substituted for Pin-b. ${ }^{3}$ 
447 The result supports the hypothesis that Pin function within wheat endosperm is lipid

448 mediated. ${ }^{33}$ In addition, the different lipid-binding behavior of these proteins provides further

449 insight into the impact of hydrophobic and cationic amino acids on the functional properties

450 of antimicrobial peptides and proteins. 


\section{REFERENCES}

452 1. Bowles, D. J.. Defense-related proteins in higher plants. Ann. Rev. Biochem. 1990, 59, 873-907.

2. Broekaert, W.F.; Cammue, B. P. A.; De Bolle, M. F. C.; Thevissen, K.; De Samblanx, G. W.; Osborn, R. W.; Nielson, K. Antimicrobial peptides from plants. Crit. Rev. Plant Sci. 1997, 16, 297-323.

3. Clifton, L. A.; Green, R. J.; Frazier, R. A. Puroindoline-b mutations control the lipid binding interactions in mixed puroindoline-a:puroindoline-b systems. Biochemistry 2007, 46, 13929-13937.

4. Clifton, L. A.; Sanders, M.; Kinane, C.; Arnold, T.; Edler, K. J.; Neylon, C.; Green, interactions: a comparison of $\alpha 1$ and $\alpha 2$-purothionin adsorbed anionic phospholipid monolayers. Phys. Chem. Chem. Phys. 2012, 14, 13569-13579.

5. Clifton, L. A.; Sanders, M. R.; Hughes, A. V.; Neylon, C.; Frazier, R. A.; Green, R. J. Lipid binding interactions of antimicrobial plant seed defence proteins: puroindoline-a and $\beta$-purothionin. Phys. Chem. Chem. Phys. 2011, 13, 17153-17162.

6. Petersen, F. N. R.; Jensen, M. O.; Nielsen, C. H. Interfacial tryptophan residues: A role for the cation-pi effect? Biophys. J. 2005, 89, 3985-3996.

7. Capparelli, R.; Palumbo, D.; Iannaccone, M.; Ventimiglia, I.; Di Salle, E.; Capuano, F.; Salvatore, P.; Amoroso, M. G. Cloning and expression of two plant proteins: similar antimicrobial activity of native and recombinant form. Biotechnol. Lett. 2006, 28, 943-949.

8. Jing, W. G.; Demcoe, A. R.; Vogel, H. J. Conformation of a bactericidal domain of puroindoline a: Structure and mechanism of action of a 13-residue antimicrobial peptide. J. Bacteriol. 2003, 185, 4938-4947. 
476 9. Dubreil, L.; Gaborit, T.; Bouchet, B.; Gallant, D. J.; Broekaert, W. F.; Quillien, L.;

477

478

479

480

481

482

483

484

485

486

487

488

489

490

491

492

493

494

495

496

497

498
Marion, D. Spatial and temporal distribution of the major isoforms of puroindolines (puroindoline-a and puroindoline-b) and non specific lipid transfer protein (nsLTPle(1)) of Triticum aestivum seeds. Relationships with their in vitro antifungal properties. Plant Sci. 1998, 138, 121-135.

10. Bhave, M.; Morris, C. F. Molecular genetics of puroindolines and related genes: regulation of expression, membrane binding properties and applications. Plant Mol. Biol. 2008, 66, 221-231.

11. Lillemo, M.; Simeone, M. C.; Morris, C. F. Analysis of puroindoline $a$ and $b$ sequences from Triticum aestivum cv. 'Penawawa' and related diploid taxa. Euphytica 2002, 126, 321-331.

12. Martin, J. M.; Frohberg, R. C.; Morris, C. F.; Talbert, L. E.; Giroux, M. J. Milling and bread baking traits associated with puroindoline sequence type in hard red spring wheat. Crop Sci. 2001, 41, 228-234.

13. Martin, J. M.; Meyer, F. D.; Morris, C. F.; Giroux, M. J. Pilot scale milling characteristics of transgenic isolines of a hard wheat over-expressing puroindolines. Crop Sci. 2007, 47, 497-506.

14. Morris, C. F.; Lillemo, M.; Simeone, M. C.; Giroux, M. J.; Babb, S. L.; Kidwell, K. K. Prevalence of puroindoline grain hardness genotypes among historically significant North American spring and winter wheats. Crop Sci. 2001, 41, 218-228.

15. Clifton, L. A.; Green, R. J.; Hughes, A. V.; Frazier, R. A. Interfacial structure of wildtype and mutant forms of puroindoline-b bound to DPPG monolayers. J. Phys. Chem. B 2008, 112, 15907-15913. 
499 16. Clifton, L. A.; Lad, M. D.; Green, R. J.; Frazier, R. A. Single amino acid substitutions in puroindoline-b mutants influence lipid binding properties. Biochemistry 2007, 46, $2260-2266$.

17. Clore, G. M.; Nilges, M.; Sukumaran, D. K.; Brunger, A. T.; Karplus, M.;

Gronenborn, A. M. The 3-dimensional structure of alpha-1-purothionin in solutioncombined use of nuclear-magnetic-resonance, distance geometry and restrained molecular dynamics. EMBO J. 1986, 5, 2729-2735.

18. Hughes, P.; Dennis, E.; Whitecross, M.; Llewellyn, D.; Gage, P. The cytotoxic plant protein, $\beta$-purothionin, forms ion channels in lipid membranes. J. Biol. Chem. 2000, $275,823-827$.

19. Llanos, P.; Henriquez, M.; Minic, J.; Elmorjani, K.; Marion, D.; Riquelme, G.; Molgo, J.; Benoit, E. Puroindoline-a and alpha 1-purothionin form ion channels in giant liposomes but exert different toxic actions on murine cells. FEBS J. 2006, 273,

20. Day, L.; Bhandari, D. G.; Greenwell, P.; Leonard, S. A.; Schofield, J. D. Characterization of wheat puroindoline proteins. FEBS J. 2006, 273, 5358-5373.

21. Jones, B. L.; Lookhart, G. L.; Johnson, D. E. Improved separation and toxicity analysis-methods for purothionins. Cereal Chem. 1985, 62, 327-331.

22. Lad, M. D.; Birembaut, F.; Clifton, L. A.; Frazier, R. A.; Webster, J. R. P.; Green, R. J. Antimicrobial peptide-lipid binding interactions and binding selectivity. Biophys. J.

520 23. Green, R. J.; Su, T. J.; Lu, J. R.; Webster, J. R. P. The displacement of preadsorbed protein with a cationic surfactant at the hydrophilic $\mathrm{SiO}_{2}$-water interface. J. Phys. Chem. B 2001, 105, 9331-9338. 
24. Wolf, M. B. A. E. Principles of Optics, 6th edition; Pergamon Press: Oxford, UK, 1984.

25. Penfold, J.; Richardson, R. M.; Zarbakhsh, A.; Webster, J. R. P.; Bucknall, D. G.; Rennie, A. R.; Jones, R. A. L.; Cosgrove, T.; Thomas, R. K.; Higgins, J. S.; Fletcher, P. D. I.; Dickinson, E.; Roser, S. J.; McLure, I. A.; Hillman, A. R.; Richards, R. W.; Staples, E. J.; Burgess, A. N.; Simister, E. A.; White, J. W. Recent advances in the study of chemical surfaces and interfaces by specular neutron reflection. J. Chem. Soc.-Faraday Trans. 1997, 93, 3899-3917.

26. Thomas, R. K. Neutron reflection from liquid interfaces. Ann. Rev. Phys. Chem. 2004, $55,391-426$.

27. Penfold, J.; Staples, E.; Thompson, L.; Tucker, I. The composition of nonionic surfactant mixtures at the air/water interface as determined by neutron reflectivity. Colloid Surf. A-Physicochem. Eng. Asp. 1995, 102, 127-132.

28. Lu, J. R.; Lee, E. M.; Thomas, R. K. The analysis and interpretation of neutron and Xray specular reflection. Acta Cryst. A 1996, 52, 11-41.

29. Richard, J. A.; Kelly, I.; Marion, D.; Auger, M.; Pezolet, M. Structure of betapurothionin in membranes: a two-dimensional infrared correlation spectroscopy study. Biochemistry 2005, 44, 52-61.

30. Mattei, C.; Elmorjani, K.; Molgo, J.; Marion, D.; Benoit, E. The wheat proteins puroindoline-a and alpha 1-purothionin induce nodal swelling in myelinated axons. Neuroreport 1998, 9, 3803-3807.

31. Branlard, G.; Amiour, N.; Igrejas, G.; Gaborit, T.; Herbette, S.; Dardevet, M.; Marion, D. Diversity of puroindolines as revealed by two-dimensional electrophoresis. Proteomics 2003, 3, 168-174. 
547 32. Clifton, L. A.; Sanders, M. R.; Castelletto, V.; Rogers, S. E.; Heenan, R. K.; Neylon, 548 C.; Frazier, R. A.; Green, R. J. Puroindoline-a, a lipid binding protein from common wheat, spontaneously forms prolate protein micelles in solution. Phys. Chem. Chem. Phys. 2011, 13, 8881-8888.

551 33. Turnbull, K. M.; Rahman, S. Endosperm texture in wheat. J. Cereal Sci. 2002, 36, 552 $327-337$.

\section{$553 \quad$ Funding}

554 We acknowledge the financial support of the Science and Technology Facilities Council for a 555 Research Network Studentship (CMSD08-02) co-funded by University of Reading and for a 556 ISIS Direct Access Beamtime Award (RB1120373).

557

558 
561 Figure 1. Surface pressure (A and C) and amide I peak areas (B and D) as a function of time

562 for co-binding of $\beta$-Pth/Pin-a (black line, triangles), $\beta$-Pth/Pin-b (black dotted line, diamonds) and $\beta$-Pth/Pin-bs (grey line, crosses) to a DPPG monolayer. Total protein concentration used is $0.48 \mu \mathrm{M}$ for $\mathrm{A}$ and $\mathrm{B}$ and $0.96 \mu \mathrm{M}$ for $\mathrm{C}$ and $\mathrm{D}$.

565

566

Figure 2. Amide spectral region showing the co-binding of (A) $\beta-P$ th/Pin-a, (B) $\beta-P$ th/Pin-b and (C) $\beta$-Pth/Pin-bs to the DPPG surface. Spectra are provided for $0,15,45$ and 60 min after addition of $0.96 \mu \mathrm{M}$ protein to the lipid subphase and presented offset with increasing adsorption time in descending order. Deconvolution of the amide I peak is also provided for 15 (bold line) and 60 (dashed line) min spectra.

Figure 3. Surface pressure (A and C) and amide I peak areas (B and D) as a function of time for sequential adsorption of proteins to a DPPG monolayer. A and B show adsorption of 0.48 $\mu \mathrm{M} \beta$-Pth followed by $0.48 \mu \mathrm{M}$ Pin-a (solid black line or triangles) or $0.48 \mu \mathrm{M}$ Pin-b (dotted black line or diamonds). $\mathrm{C}$ and $\mathrm{D}$ show adsorption of $0.48 \mu \mathrm{M}$ Pin-a (solid black line or triangles) or $0.48 \mu \mathrm{M}$ Pin-b (dotted black line or diamonds) followed by $0.48 \mu \mathrm{M} \beta$-Pth. The arrows indication the time points for addition of protein to the subphase. The total protein concentration added for each experiment is $0.96 \mu \mathrm{M}$.

Figure 4. Amide I spectra showing the sequential adsorption to DPPG monolayer for $\beta$-Pth

582 Each panel shows three spectra; before protein addition (top), 130 min after addition of first protein (middle) and approximately 100 min after addition of the second protein (bottom). 
585 Figure 5. (A) The neutron reflectivity profile for chain deuterated DPPG at the air/water

586 interface showing best two-layer model-to-data fit as the solid line. (B) The scattering length

587 density profile as a function of distance from interface as determined from the fit. The

588 corresponding fit parameters are provided in Table 5.

589

590 Figure 6. (A) The neutron reflectivity profile for chain deuterated and hydrogenated DPPG

591 with co-adsorbed $0.48 \mu \mathrm{M} \beta$-Pth/Pin-a showing best two-layer model-to-data fit (grey line for

592 h-lipid contrast and black line for d-lipid contrast). (B) The corresponding scattering length

593 density profile as a function of distance from interface. (C) The neutron reflectivity profile

594 for chain deuterated and hydrogenated DPPG with sequential adsorbed $0.96 \mu \mathrm{M}$ total

595 concentration $\beta$-Pth/Pin-a showing best two-layer model-to-data fit (grey line for h-lipid

596 contrast and black line for d-lipid contrast). (D) The corresponding scattering length density

597 profile as a function of distance from interface.

598 


\section{TABLES}

Table 1. Summary of Scattering Length, Scattering Length Density and Molecular Mass of the Hydrogenated (h) and Deuterated (d) Lipid and Protein Components

\begin{tabular}{|c|c|c|c|}
\hline Lipid/ Protein & $\begin{array}{l}\text { Scattering length } \\
\qquad\left(10^{-3} \AA\right)\end{array}$ & $\begin{array}{l}\text { Scattering length } \\
\text { density }\left(10^{-6} / \AA^{2}\right)\end{array}$ & $\begin{array}{c}\text { Molecular weight } \\
\text { (g/mol) }\end{array}$ \\
\hline (h) DPPG & 0.38 & 0.36 & 721 \\
\hline (tail d) DPPG & 6.84 & 6.24 & 783 \\
\hline DPPG headgroup & 6.13 & 2.52 & 299 \\
\hline (h) DPPG tail region & -0.32 & -0.398 & 422 \\
\hline (d) DPPG tail region & 6.13 & 7.54 & 484 \\
\hline Pin-a in NRW & 31.13 & 1.97 & 12290 \\
\hline$\beta$-Pth in NRW & 11.19 & 1.86 & 4953 \\
\hline
\end{tabular}


Table 2. Change in Surface Pressure $(\Delta \pi)$ and Amide I Peak Areas for Co-adsorption of Puroindolines and $\beta$-Pth to a Condensed Phase DPPG Layer

\begin{tabular}{|c|c|c|c|}
\hline $\begin{array}{l}\text { Protein concentration } \\
\qquad(\mu \mathrm{M})\end{array}$ & Protein mix & $\Delta \pi(\mathrm{mN} / \mathrm{m})$ & $\begin{array}{c}\text { Amide I peak } \\
\text { area }\end{array}$ \\
\hline 0.48 & $\beta$-Pth & $9.5 \pm 0.6$ & $0.028 \pm 0.006$ \\
\hline 0.48 & Pin-a & $7.1 \pm 1.0$ & $0.132 \pm 0.008$ \\
\hline 0.48 & Pin-b & $9.7 \pm 0.7$ & $0.095 \pm 0.009$ \\
\hline 0.48 & Pin-bs & $6.1 \pm 0.7$ & $0.105 \pm 0.005$ \\
\hline 0.48 & $\beta$-Pth/Pin-a & $7.3 \pm 0.8$ & $0.058 \pm 0.008$ \\
\hline 0.48 & $\beta-P$ th/Pin-b & $3.2 \pm 0.3$ & $0.043 \pm 0.014$ \\
\hline 0.48 & $\beta$-Pth/Pin-bs & $7.9 \pm 0.7$ & $0.017 \pm 0.011$ \\
\hline 0.96 & $\beta$-Pth/Pin-a & $9.9 \pm 0.6$ & $0.135 \pm 0.004$ \\
\hline 0.96 & $\beta$-Pth/Pin-b & $11.3 \pm 0.5$ & $0.101 \pm 0.017$ \\
\hline 0.96 & $\beta$-Pth/Pin-bs & $13.7 \pm 0.7$ & $0.112 \pm 0.009$ \\
\hline
\end{tabular}


Table 3. Change in Surface Pressure $(\Delta \pi)$ During Sequential Protein Addition to Condensed Phase DPPG Monolayers

\begin{tabular}{lccc}
\hline Sequential & First protein & Second protein & Total \\
adsorption of: & $\Delta \boldsymbol{\pi}(\mathbf{m N} / \mathbf{m})$ & $\Delta \boldsymbol{\pi}(\mathbf{m} \mathbf{N} / \mathbf{m})$ & $\Delta \boldsymbol{\pi}(\mathbf{m} \mathbf{N} / \mathbf{m})$ \\
\hline $0.48 \mu \mathrm{M} \beta$-Pth then & $9.5 \pm 0.6$ & $1.6 \pm 0.3$ & $11.1 \pm 0.4$ \\
$0.48 \mu \mathrm{M}$ Pin-a & & $1.5 \pm 1$ & \\
\hline $0.48 \mu \mathrm{M}$ Pin-a then & $7.9 \pm 1$ & $9.3 \pm 0.3$ \\
$0.48 \mu \mathrm{M} \beta$-Pth & & $0.4 \pm 0.3$ & \\
\hline $0.48 \mu \mathrm{M} \beta$-Pth then & $9.0 \pm 0.8$ & & $10.4 \pm 0.5$ \\
$0.48 \mu \mathrm{M}$ Pin-b & & $1 \pm 0.6$ & \\
\hline $0.48 \mu \mathrm{M}$ Pin-b then & $9.2 \pm 0.7$ & & \\
$0.48 \mu \mathrm{M} \beta$-Pth & & & \\
\hline
\end{tabular}


Table 4. Change in ER-FTIR Amide I Peak Area During Sequential Protein Addition to Condensed Phase DPPG Monolayers

\begin{tabular}{|c|c|c|}
\hline Sequential adsorption of: & $\begin{array}{l}\text { Amide I peak Area after } \\
\text { addition of } 1^{\text {st }} \text { protein }\end{array}$ & $\begin{array}{l}\text { Amide I peak area after } \\
\text { addition of } 2^{\text {nd }} \text { protein }\end{array}$ \\
\hline $\begin{array}{l}0.48 \mu \mathrm{M} \beta \text {-Pth } \\
\text { then } 0.48 \mu \mathrm{M} \text { Pin-a }\end{array}$ & $0.028 \pm 0.005$ & $0.128 \pm 0.009$ \\
\hline $\begin{array}{l}0.48 \mu \mathrm{M} \text { Pin-a } \\
\text { then } 0.48 \mu \mathrm{M} \beta \text {-Pth }\end{array}$ & $0.132 \pm 0.012$ & $0.135 \pm 0.013$ \\
\hline $\begin{array}{l}0.48 \mu \mathrm{M} \beta \text {-Pth } \\
\text { then } 0.48 \mu \mathrm{M} \text { Pin-b }\end{array}$ & $0.028 \pm 0.005$ & $0.075 \pm 0.011$ \\
\hline $\begin{array}{l}0.48 \mu \mathrm{M} \text { Pin-b } \\
\text { then } 0.48 \mu \mathrm{M} \beta \text {-Pth }\end{array}$ & $0.075 \pm 0.013$ & $0.093 \pm 0.009$ \\
\hline
\end{tabular}


Table 5. NR Fit Parameters for Pin-a/ $\beta$-Pth Binding to DPPG

\begin{tabular}{|c|c|c|c|c|c|c|}
\hline \multirow[b]{2}{*}{ Layer $+H / D$ contrast } & \multicolumn{2}{|c|}{ Fit Parameters } & \multirow[b]{2}{*}{$\Phi_{\text {lipid }}$} & \multirow[b]{2}{*}{$\boldsymbol{\Phi}_{\text {protein }}$} & \multirow[b]{2}{*}{$\begin{array}{l}\mathbf{A}_{\text {lipid }} \\
\left(\AA^{2}\right)\end{array}$} & \multirow[b]{2}{*}{$\begin{array}{c}\Gamma_{\text {prot }} \\
\left(\mathrm{mg} / \mathrm{m}^{2}\right)\end{array}$} \\
\hline & $\begin{array}{c}\tau \\
(\AA)\end{array}$ & $\begin{array}{c}\rho \\
\left(10^{-6} / \AA^{2}\right)\end{array}$ & & & & \\
\hline \multicolumn{7}{|l|}{ DPPG only } \\
\hline \multicolumn{7}{|l|}{ Layer 1} \\
\hline d-DPPG on NRW & 16.4 & 6.9 & 0.91 & - & 54.1 & - \\
\hline \multicolumn{7}{|l|}{ Layer 2} \\
\hline d-DPPG on NRW & 6.3 & 2.3 & 0.91 & - & 49.3 & - \\
\hline \multicolumn{7}{|c|}{$0.96 \mu \mathrm{M}$ Pin-a/ $\beta$-Pth co-adsorbed to DPPG } \\
\hline \multicolumn{7}{|l|}{ Layer 1} \\
\hline d-DPPG on NRW & 17.3 & 6.4 & 0.81 & 0.16 & 58.2 & 0.32 \\
\hline h-DPPG on NRW & 17.3 & -0.01 & & & & \\
\hline \multicolumn{7}{|l|}{ Layer 2} \\
\hline d-DPPG on NRW & 8 & 1.79 & 0.61 & 0.13 & 58.2 & 0.14 \\
\hline h-DPPG on NRW & 8 & 1.79 & & & & \\
\hline \multicolumn{7}{|l|}{ Layer 3} \\
\hline d-DPPG on NRW & 37 & 0.7 & - & 0.36 & - & 1.72 \\
\hline h-DPPG on NRW & 37 & 0.7 & & & & \\
\hline \multicolumn{7}{|c|}{$0.48 \mu \mathrm{M} \beta$-Pth adsorbed to $0.48 \mu \mathrm{M}$ Pin-a and DPPG } \\
\hline \multicolumn{7}{|l|}{ Layer 1} \\
\hline d-DPPG on NRW & 20 & 4.1 & 0.5 & 0.16 & 81.0 & 0.45 \\
\hline h-DPPG on NRW & 20 & 0.15 & & & & \\
\hline \multicolumn{7}{|l|}{ Layer 2} \\
\hline d-DPPG on NRW & 10 & 1.65 & 0.39 & 0.35 & 81.0 & 0.51 \\
\hline h-DPPG on NRW & 10 & 1.65 & & & & \\
\hline \multicolumn{7}{|l|}{ Layer 3} \\
\hline d-DPPG on NRW & 34 & 0.8 & - & 0.41 & - & 1.81 \\
\hline h-DPPG on NRW & 34 & 0.8 & & & & \\
\hline
\end{tabular}


Figure 1
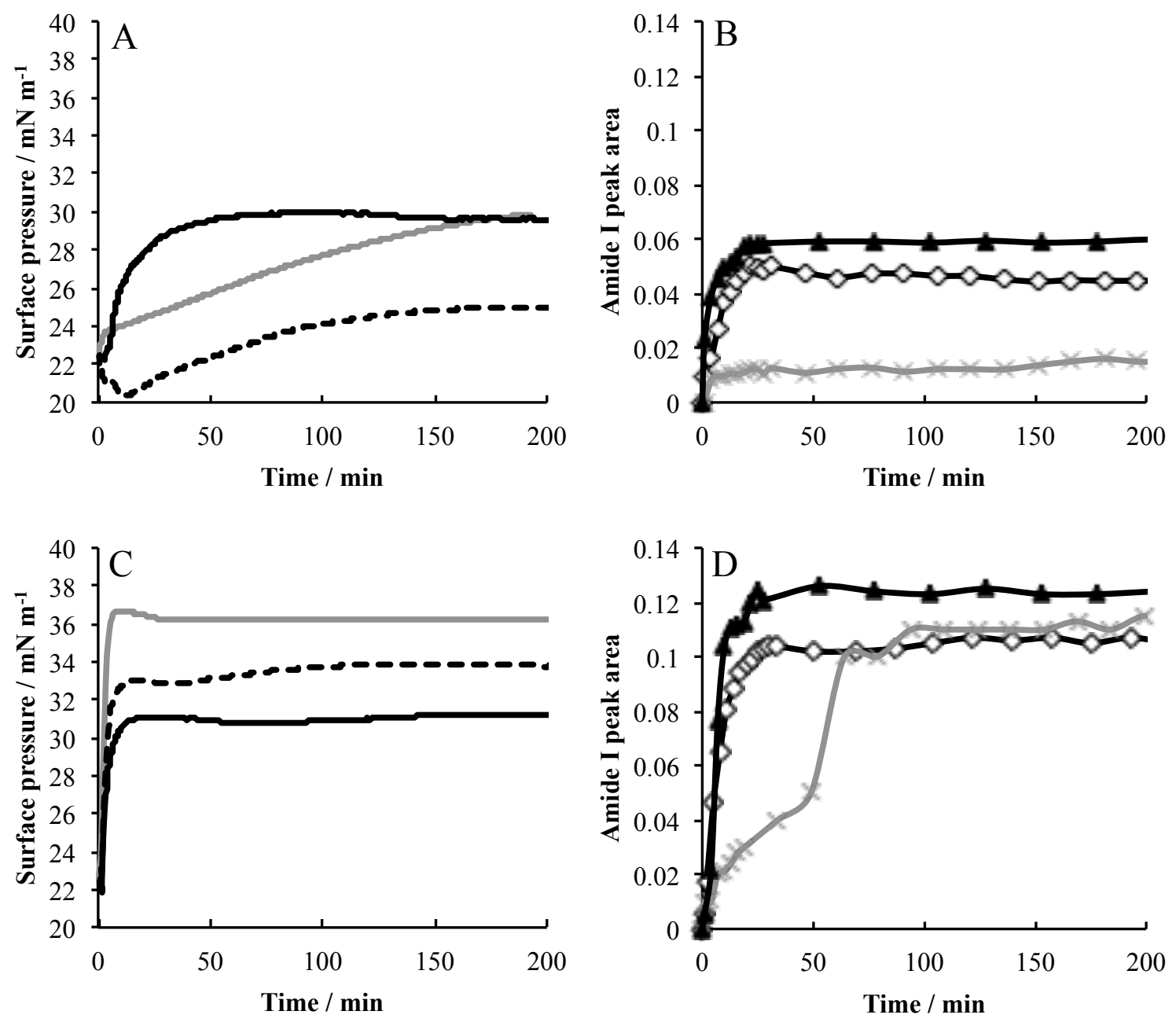
Figure 2
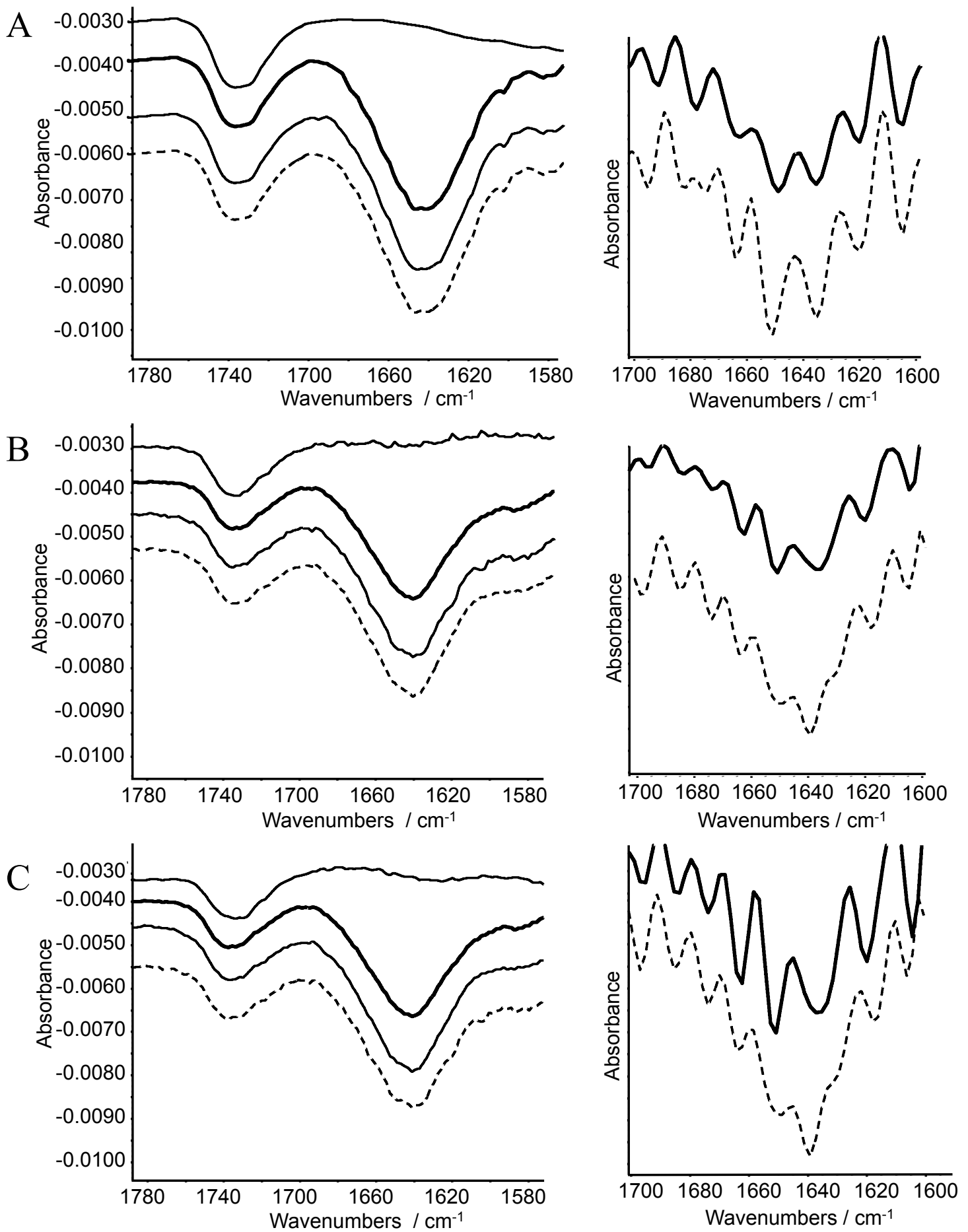
Figure 3
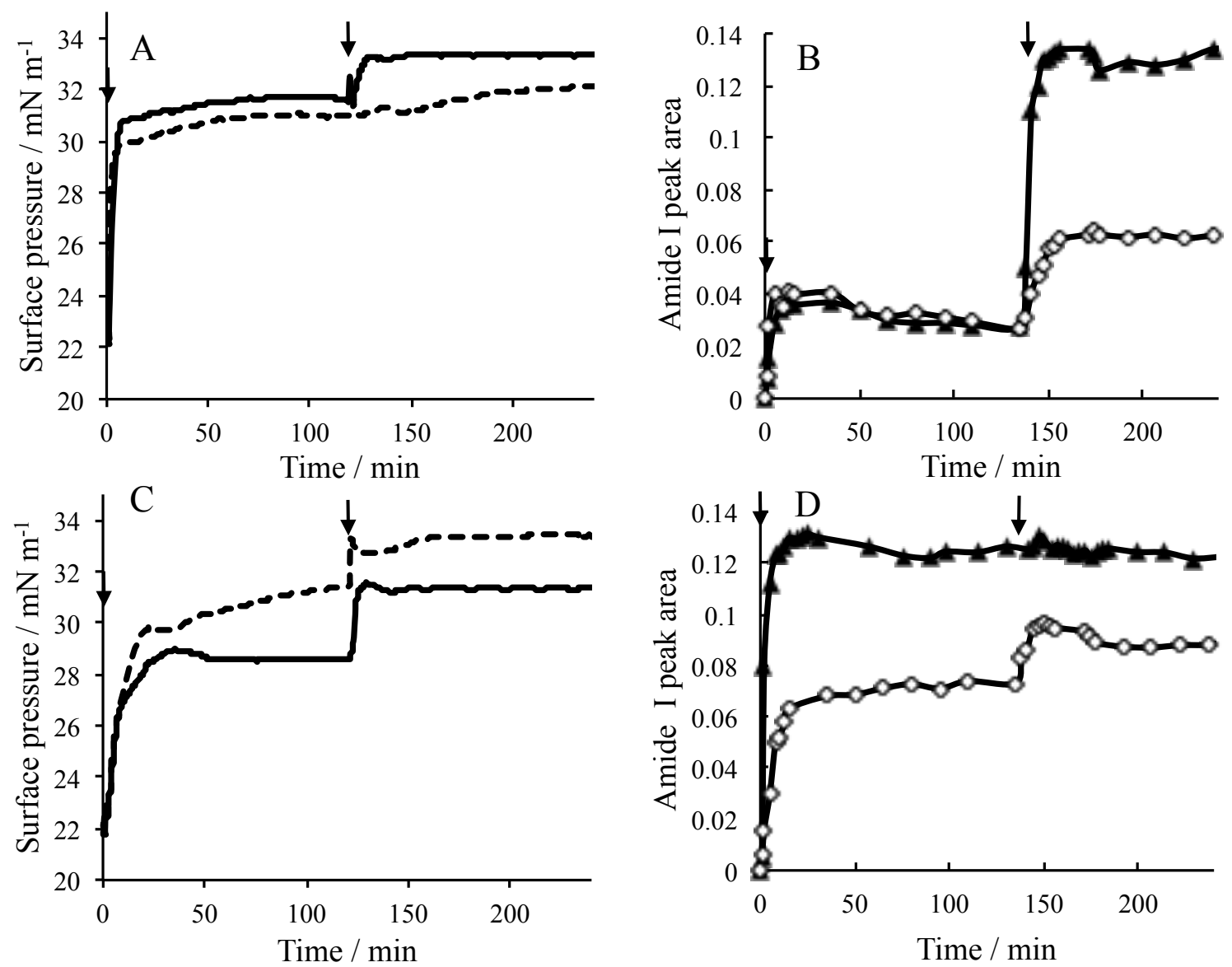
Figure 4

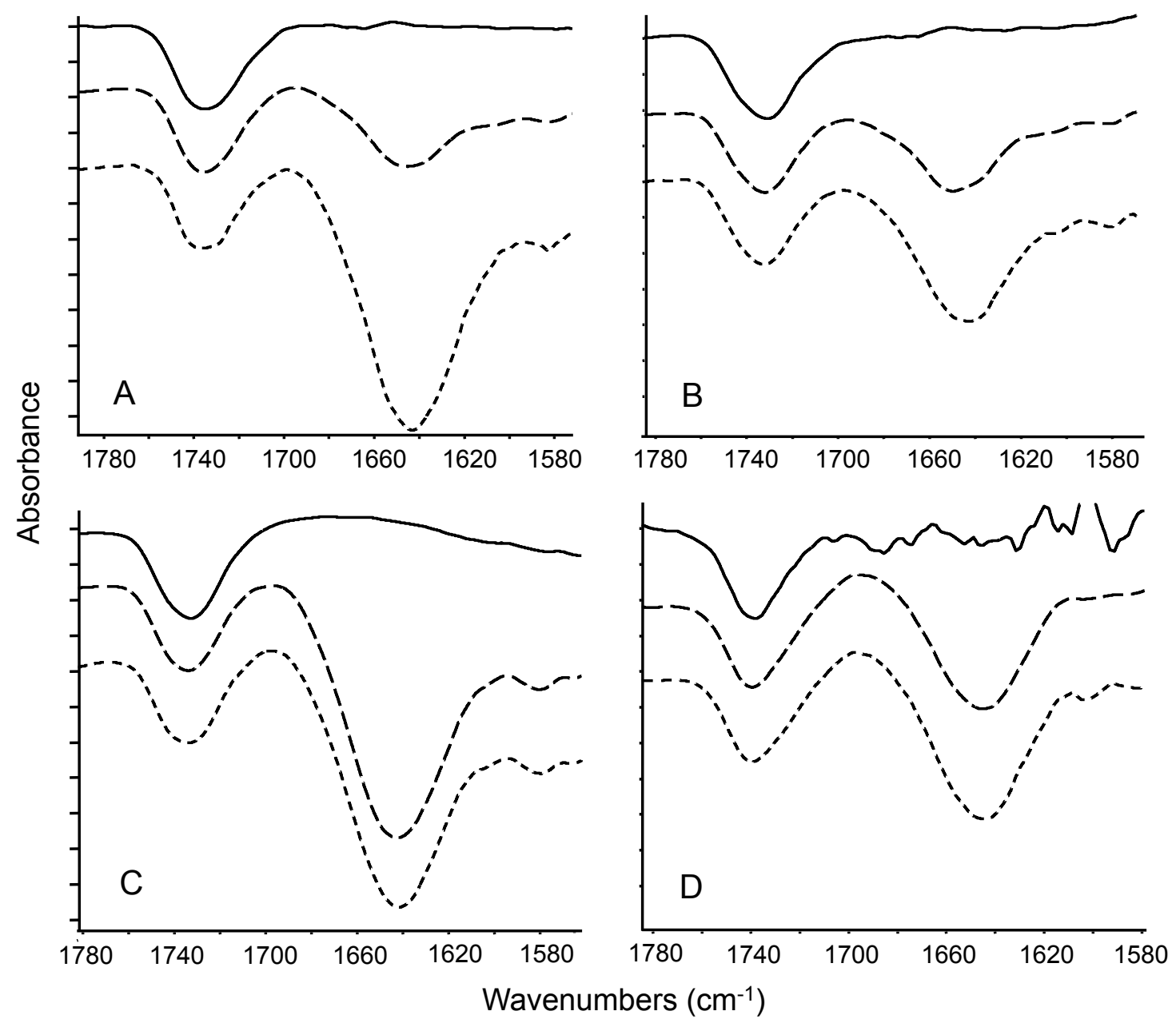


Figure 5
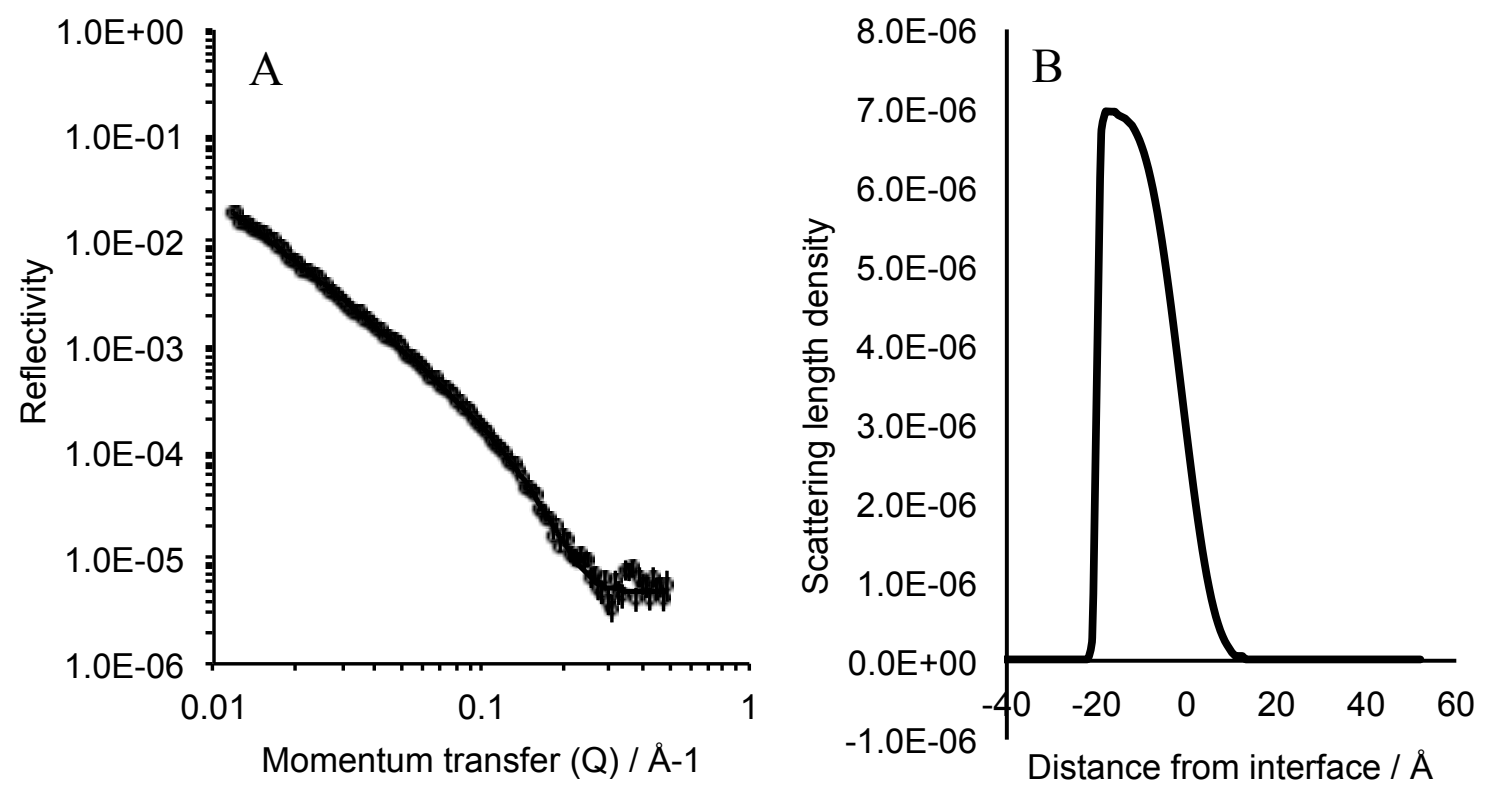
Figure 6
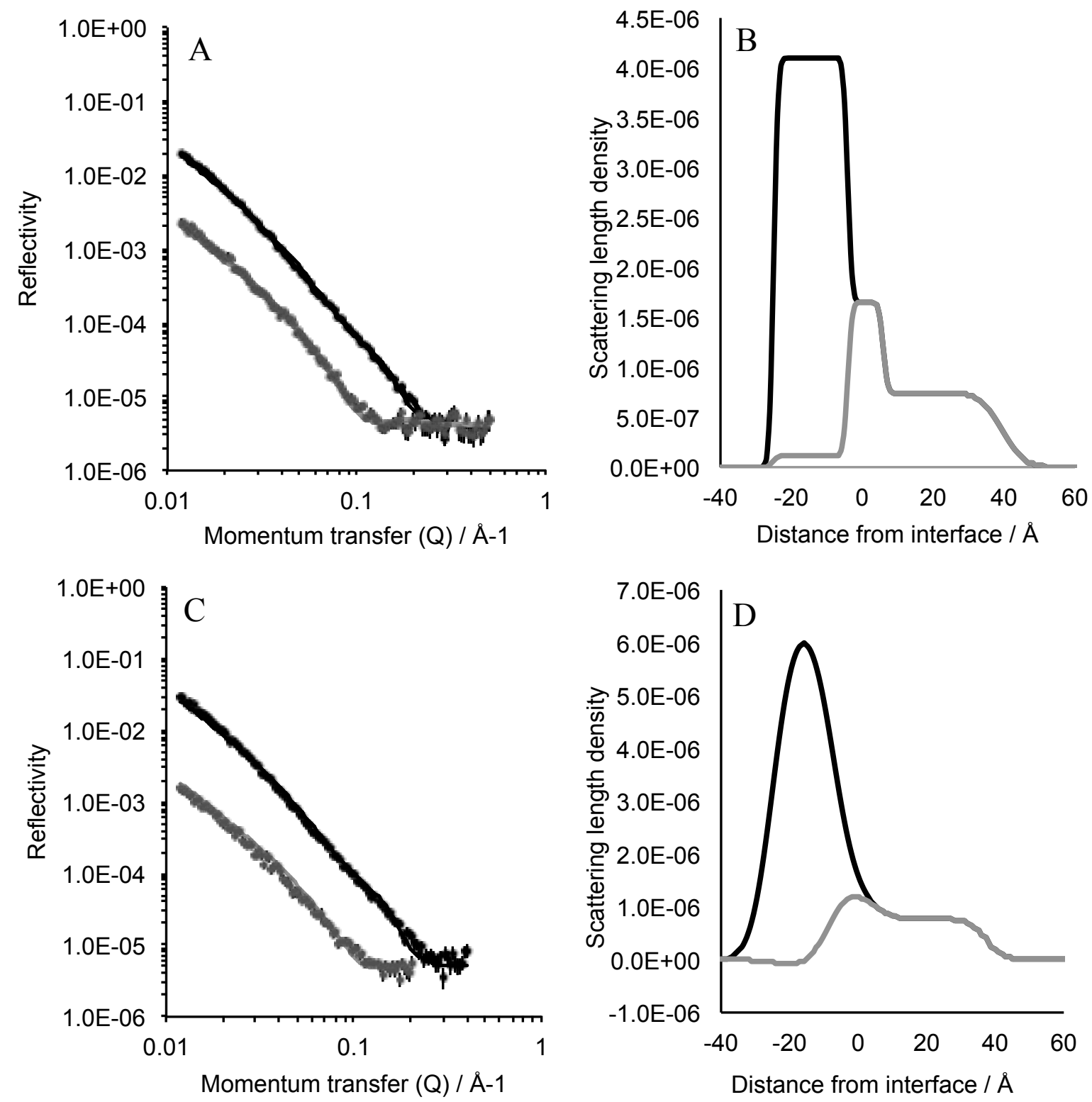


\section{TOC Graphic}
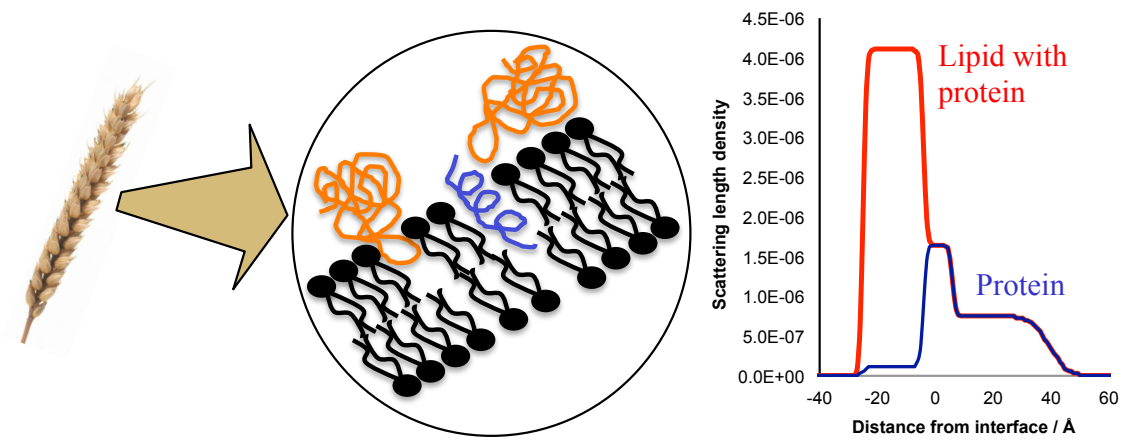